\title{
On the Added Value of Baseline FDG-PET in Malignant Lymphoma
}

\author{
Henriette Quarles van Ufford, ${ }^{1,2}$ Otto Hoekstra, ${ }^{3}$ Marie de Haas, ${ }^{4}$ Rob Fijnheer, ${ }^{5}$ \\ Shulamiet Wittebol, ${ }^{5}$ Bianca Tieks, ${ }^{4}$ Mark Kramer, ${ }^{6}$ John de Klerk ${ }^{4}$ \\ ${ }^{1}$ Radiology, University Medical Center Utrecht, Utrecht, The Netherlands \\ ${ }^{2}$ Radiology and Nuclear Medicine, Meander Medical Center, Utrecht, The Netherlands \\ ${ }^{3}$ Nuclear Medicine \& PET research, Vrije University Medical Center, Amsterdam, The Netherlands \\ ${ }^{4}$ Nuclear Medicine, Meander Medical Center, Amersfoort, The Netherlands \\ ${ }^{5}$ Internal Medicine, Meander Medical Center, Amersfoort, The Netherlands \\ ${ }^{6}$ Internal Medicine, Vrije University Medical Centre, Amsterdam, The Netherlands
}

\begin{abstract}
Purpose: The added value of baseline positron emission tomography (PET) scans in therapy evaluation in malignant lymphoma is unclear. In guidelines, baseline PET is recommended but not mandatory except in lymphoma types with variable fluoro-D-glucose uptake. The aim of the present study was to test the hypothesis that adding baseline PET information decreases false positive readings with posttreatment PET and improves observer agreement.

Methods: Forty-four patients (mean age 56 years, standard deviation 14) with malignant lymphoma were included. Two nuclear medicine physicians retrospectively and independently evaluated the posttreatment PET, 3 weeks later followed by paired reading of baseline and posttreatment PET. For each PET, 22 regions were classified as positive, negative, or equivocal, resulting in an overall PET score of positive, unclear, or negative. In case of discrepancies, consensus was reached.

Results: Addition of baseline to posttreatment PET evaluation affected the classification of metabolic response in $34 \%$ of malignant lymphoma patients treated with first-line chemotherapy. In one out of seven patients, addition of the baseline PET lead to opposite conclusions (95\% confidence interval 4-14). False positivity was reduced by adding the baseline scan information, but the effect on false negativity was similar. In addition, the amount of unclear classifications halved after paired reading. Observer agreement did not improve upon adding the baseline PET data. Conclusion: Without any other clinical information, pretreatment PET facilitates changes the interpretation of a posttreatment PET in a third of the patients, resulting in both upgrading and downgrading of the posttreatment situation of a malignant lymphoma patient. If these results are confirmed for PET-computed tomography systems, they favor the addition of baseline PET to the current work-up of patients with malignant lymphoma.
\end{abstract}

Key words: FDG PET, Malignant lymphoma, Therapy evaluation, Baseline PET, Hodgkin's disease, Non-Hodgkin's lymphoma

\section{Introduction}

$\mathrm{P}$ rognosis and survival of patients with Hodgkin's disease (HD) and non-Hodgkin's lymphoma (NHL) depend on

Correspondence to: Henriette Quarles van Ufford; e-mail: h.m.e.quarlesv anufford@umcutrecht.nl both histological grade and clinical stage at the moment of diagnosis and furthermore on the response to treatment [1]. The Ann Arbor classification with Cotswolds revision is the guideline for radiological staging of $\mathrm{HD}$ and most types of NHL $[2,3]$. In the past, the Ann Arbor staging system was based on physical examination and bone marrow evaluation, later to include the computed tomography (CT) scan. With the 
Table 1. Patient characteristics of NHL and HD patients

\begin{tabular}{lll}
\hline Patient & NHL & HD \\
\hline Number & 35 & 9 \\
M:F & $16: 19$ & $7: 2$ \\
Mean age (years; range) & $58(24-87)$ & $40(19-71)$ \\
Pathological subtypes & DLBCL, 18 & Nodular sclerosis, 7 \\
& Follicular, 9 & Mixed cellularity, 2 \\
& Burkitt, 1 & \\
& MCL, 2 & \\
MZL, 1 & \\
& MALT, 1 & \\
& SLL, 2 & \\
& ACL, 1 & \\
\end{tabular}

NHL non-Hodgkin's lymphoma, $H D$ Hodgkin's disease, $M$ male, $F$ female, $D L B C L$ diffuse large B cell lymphoma, $M C L$ mantle cell lymphoma, $M Z L$ marginal zone lymphoma, MALT mucosa-associated lymphoid tissue, $S L L$ small lymphocytic lymphoma, $A C L$ anaplastic cell lymphoma

implementation of the positron emission tomography (PET) scan and later the integrated PET/CT scan, several studies concluded that a baseline 2-deoxy-2-[18F]fluoro-D-glucose (FDG) PET scan provides significant more information than conventional $\mathrm{CT}$, with subsequent therapeutic consequences [4-6]. Whereas the application of PET in therapy evaluation in malignant lymphoma is rapidly emerging, the added value of baseline PET scans in this context is less clear. In guidelines, baseline PET is recommended but not mandatory except in lymphoma types with variable FDG uptake. Baseline scans may facilitate interpretation of PET at therapy evaluation, increase reader confidence, and perhaps avoid misinterpreta- tion. Guidelines on PET reading after therapy refer to "previously involved lesions" to avoid false positivity [7-9].

The aim of the present study was to test the hypothesis that adding baseline PET information decreases false positive readings with therapy evaluation PET and improves observer agreement.

\section{Materials and Methods}

\section{Subjects}

We retrospectively studied baseline and posttreatment PET scans of 44 patients with newly diagnosed malignant lymphoma undergoing firstline therapy between January 2005 and July 2007. In all patients, malignant lymphoma had been histopathologically proven, and initial staging was done according to the Ann Arbor classification (with supplementary baseline PET scan). The posttreatment PET scan was performed at least 4-6 weeks after completion of therapy, and patients were at least 10 days off granulocyte colony-stimulating factor (GCSF) therapy $[7,10]$. The study was approved by the ethical board, and informed consent was waived.

\section{PET Imaging}

PET was performed with a mobile scanner (ECAT-ACCEL, Siemens/CTI, Knoxville, TN, USA). Patients fasted for $6 \mathrm{~h}$ before the scan. Prior to injection, blood glucose levels were within the normal range $(<11 \mathrm{mmol} / \mathrm{L})$. One hour after injection of FDG ( $5 \mathrm{MBq} / \mathrm{kg}$ body weight), patients were scanned from mid femur to

Table 2. Agreement of isolated posttreatment and reference PET classification (a), as a function of PET positivity criteria (b), and observer agreement (c)

(a)

Consensus-isolated posttreatment PET

(b)

Conservative: consensus-isolated posttreatment PET

Sensitive: consensus-isolated posttreatment PET

(c)

Observer 1 isolated posttreatment PET

Observer 2 isolated posttreatment PET
Consensus-paired reading (reference standard)

$\begin{array}{lllll} & \text { Negative } & \text { Unclear } & \text { Positive } & \text { Total } \\ \text { Negative } & 19 & 2 & 3 & 24 \\ \text { Unclear } & 3 & 0 & 3 & 6 \\ \text { Positive } & 3 & 1 & 10 & 14 \\ \text { Total } & 25 & 3 & 16 & 44\end{array}$

Conservative: consensus-paired reading (reference standard)

\begin{tabular}{llll} 
& Negative & Positive & Total \\
Negative & 24 & 6 & 30 \\
Positive & 4 & 10 & 14 \\
Total & 28 & 16 & 44 \\
Sensitive: consensus-paired reading (reference standard) & & \\
\multicolumn{2}{l}{ Negative } & Positive & Total \\
Negative & 19 & 5 & 24 \\
Positive & 6 & 14 & 20 \\
Total & 25 & 19 & 44
\end{tabular}

Observer 1 posttreatment PET (paired reading)

\begin{tabular}{lllll} 
& Negative & Unclear & Positive & Total \\
Negative & 20 & 0 & 3 & 23 \\
Unclear & 4 & 1 & 2 & 7 \\
Positive & 4 & 2 & 8 & 14 \\
Total & 28 & 3 & 13 & 44 \\
Observer & 2 posttreatment PET (paired reading) & & \\
\multicolumn{5}{r}{} \\
Negative & Negative & Unclear & Positive & Total \\
Unclear & 17 & 2 & 2 & 21 \\
Positive & 5 & 1 & 3 & 9 \\
Total & 0 & 3 & 11 & 14 \\
& 22 & 6 & 16 & 44
\end{tabular}



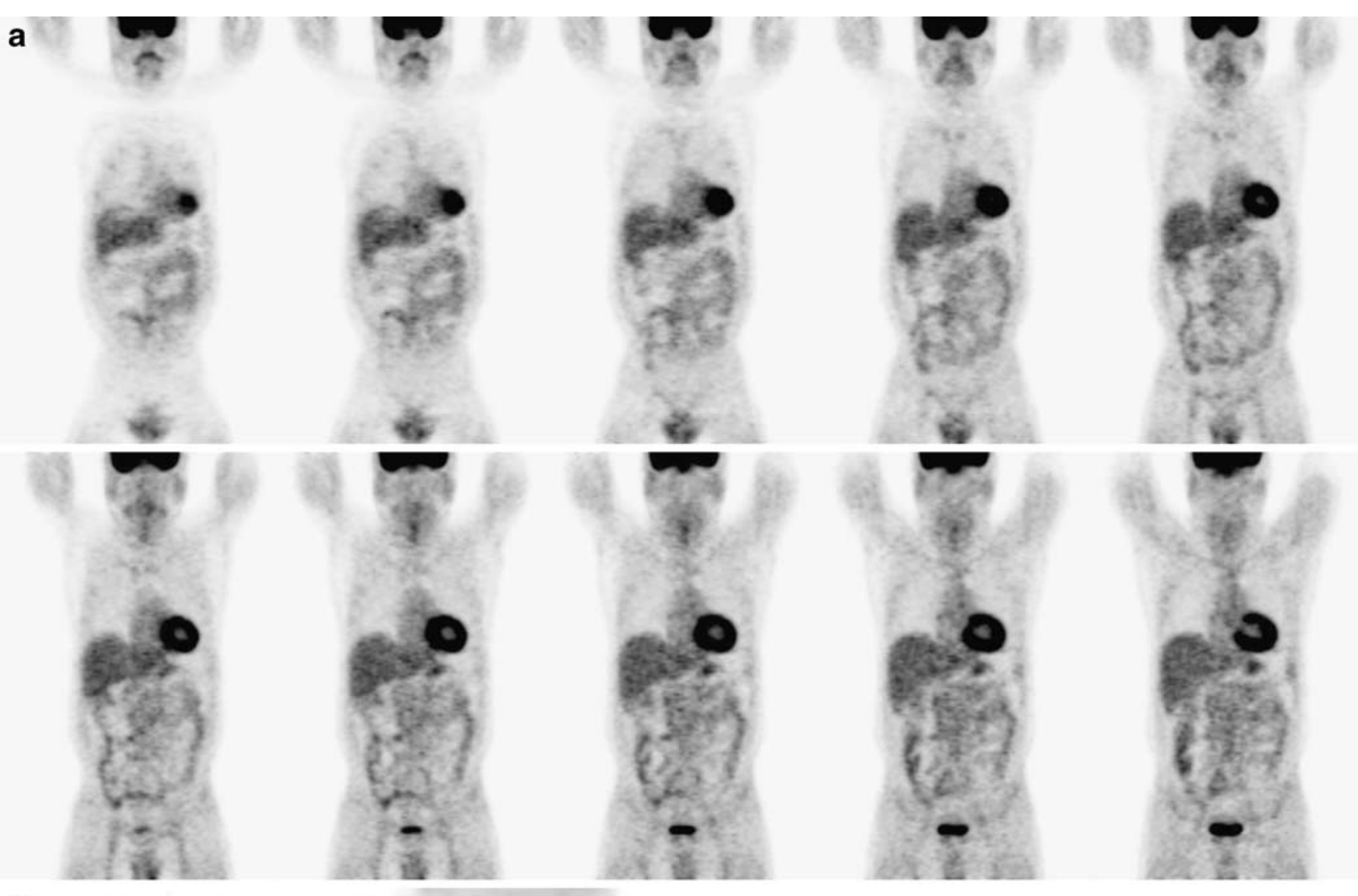

b

C
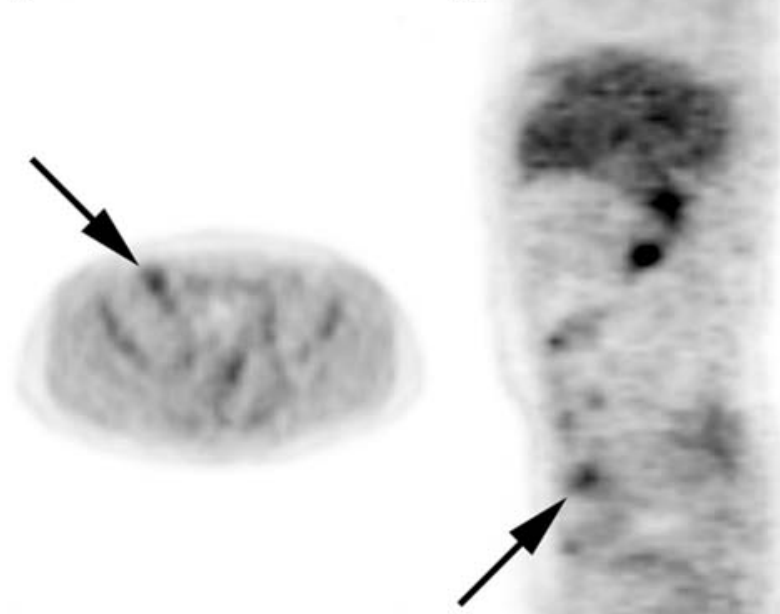

Fig. 1. Posttreatment and pretreatment PET scan of a 51-year-old male with follicular non-Hodgkin lymphoma. Both interpreters scored complete metabolic response on the isolated posttreatment PET scan. a Posttreatment PET scan: coronal images with an b axial and $\mathbf{c}$ sagittal image at the level of the described lesion. $\mathbf{d}$ Pretreatment PET scan: coronal images with an $\mathbf{e}$ axial and $\mathbf{f}$ sagittal image at the level of the described lesion. The pretreatment PET scan displays multiple regions with pathologic FDG uptake: multiple regions above (neck and mediastinum) and below (intra abdominal and inguinal) diaphragm and diffuse bone marrow involvement. With this knowledge, the initial interpretation of the posttreatment PET scan was changed to a positive posttreatment PET scan: In the right inguinal region, there is increased FDG uptake. Without the pretreatment PET scan, this was considered physiologic.

the base of the skull. Acquisition time was 5 min per bed position, with a transmission time of $60 \mathrm{~s}$ each. Patients were scanned in seven bed positions. PET images were reconstructed with and without attenuation correction using a weighted iterative ordered subsets expectation maximization algorithm (OSEM, two iterations, eight subsets). In the final step, a three-dimensional isotropic gauss filter was applied to a final image resolution with $8-9-\mathrm{mm}$ full width at half maximum. 

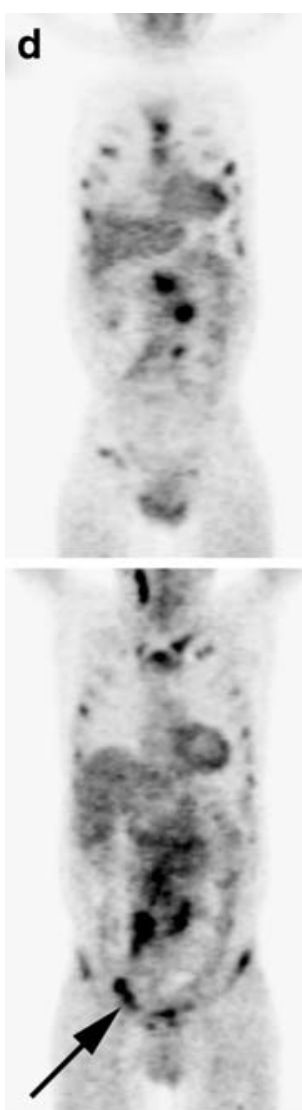

e

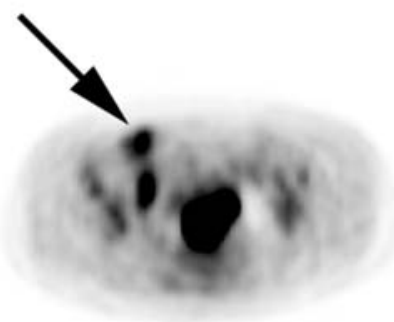

Fig. 1. (continued).

\section{PET Analysis}

Two nuclear medicine physicians with $>5$-year clinical PET experience (MJH, JMHK) independently evaluated the PET scans. First, they interpreted all 44 posttreatment PET scans, 3 weeks later followed by another session in which the same posttreatment PET scans were presented together with the baseline PET scans (paired reading). The observers were aware that the patients had malignant lymphoma but unaware of the type and grade of lymphoma and results of baseline or posttreatment conventional staging. For each scan, they analyzed 22 regions using a standardized form, classifying FDG uptake as positive, negative, or equivocal. Criteria for PET positivity were as follows: A region with FDG uptake above background in a location incompatible with normal anatomy or physiology was considered positive [7].

After evaluation of the forms of the posttreatment PET scans in both sessions, the observers were requested to assign a consensus score in case of discrepancies. Consensus was reached for the 
regions of the posttreatment PET without presentation of the baseline PET, followed by the posttreatment PET in combination with the baseline PET.

Besides this lesion-based analysis, the observers provided per patient evaluations. In each patient, all scores of the 22 different regions were taken together resulting in a negative posttreatment PET scan (all regions negative, indicating complete metabolic remission), or a positive posttreatment PET scan $(\geq 1$ positive region). Patients with only equivocal scores, besides negative scores, were classified as unclear.

Because both observers evaluated the PET scans without clinical information, the positive or equivocal classified regions of the posttreatment PET scans were compared with the initially affected regions reported in the clinical database (baseline CT, PET scan, bone marrow biopsy).

\section{Statistical Analysis}

We considered the consensus scores of the paired reading as the gold standard for presence or absence of viable tumor after therapy. To measure the impact of adding a baseline PET scan as a function of sensitivity of PET readings, the posttreatment PET results (isolated and paired reading) were dichotomized by assigning the unclear classification to either the PET-negative (complete responders) or to the PET-positive classification. Sensitivity, specificity, positive predictive value, and negative predictive value were determined for either strategy. To analyze interobserver variability and agreement of isolated and combined baseline and posttreatment PET readings, we used linear-weighted kappa $\left(\kappa_{\mathrm{w}}\right.$; SAS
9.1; SAS Institute, Cary, NC), considering kappa $<0.20$ as poor observer agreement, $021-0.40$ as fair, $0.41-0.60$ as moderate, $0.61-0.80$ as good, and $0.81-1.00$ as very good [11]. Ninety-five percent confidence intervals of proportions were calculated with Confidence Interval Analysis 2.1.2.

\section{Results}

\section{Subjects}

We included 44 patients with a mean age of 56 years (SD 14), diagnosed with NHL in $35(80 \%)$, and HD in nine $(20 \%)$ patients, further details are provided in Table 1. All patients had FDG avid lymphoma at baseline and had been clinically staged (including PET) according to the Ann Arbor classification as stage $1(n=3,7 \%)$, stage $2(n=15$, $34 \%)$, stage $3(n=16,36 \%)$, and stage $4(n=10,23 \%)$.

\section{Per Patient Analysis}

For each patient, the initial stage of disease and type of lymphoma as reported in the clinical digital database, the scores of the "isolated" posttreatment PET scans, and the paired posttreatment PET readings for either observer or their consensus scores are provided in Appendix (Table 4).

In the consensus reading of the "isolated" posttreatment PET scans, 24 PET scans were classified as negative, six as unclear,

Table 3. Per region analysis of the agreement of isolated posttreatment and reference PET classifications (a), as a function of PET positivity criteria (b), and observer agreement (c)

(a)

Consensus-isolated posttreatment PET

(b)

Conservative: consensus-isolated posttreatment PET

Sensitive: consensus-isolated posttreatment PET

(c)

Observer 1 isolated posttreatment PET

Observer 2 isolated posttreatment PET
Consensus-paired reading (reference standard)

$\begin{array}{lllll} & \text { Negative } & \text { Unclear } & \text { Positive } & \text { Total } \\ \text { Negative } & 909 & 5 & 9 & 923 \\ \text { Unclear } & 6 & 1 & 3 & 10 \\ \text { Positive } & 5 & 1 & 29 & 35 \\ \text { Total } & 920 & 7 & 41 & 968\end{array}$

Conservative: consensus-paired reading (reference standard)

\begin{tabular}{|c|c|c|c|}
\hline & Negative & Positive & Total \\
\hline Negative & 921 & 12 & 933 \\
\hline Positive & 6 & 29 & 35 \\
\hline Total & 927 & 41 & 968 \\
\hline \multicolumn{4}{|c|}{ Sensitive: consensus-paired reading (reference standard) } \\
\hline & Negative & Positive & Total \\
\hline Negative & 909 & 14 & 923 \\
\hline Positive & 11 & 34 & 45 \\
\hline Total & 920 & 48 & 968 \\
\hline
\end{tabular}

Observer 1 posttreatment PET (paired reading)

\begin{tabular}{|c|c|c|c|c|}
\hline & Negative & Unclear & Positive & Total \\
\hline Negative & 902 & 3 & 12 & 917 \\
\hline Unclear & 9 & 1 & 3 & 13 \\
\hline Positive & 17 & 2 & 19 & 38 \\
\hline Total & 928 & 6 & 34 & 968 \\
\hline \multicolumn{5}{|c|}{ Observer 2 posttreatment PET (paired reading) } \\
\hline & Negative & Unclear & Positive & Total \\
\hline Negative & 898 & 9 & 6 & 913 \\
\hline Unclear & 6 & 2 & 5 & 13 \\
\hline Positive & 10 & 2 & 30 & 42 \\
\hline Total & 914 & 13 & 41 & 968 \\
\hline
\end{tabular}


and 14 as positive. In the consensus reading of the paired reading, 25 posttherapy PET scans were classified as negative, three as unclear, and 16 as positive (Table 2). The consensus scores of the isolated posttreatment PET scans vs paired reference standard were concordant in $29 / 44$ cases $(66 \%, 95 \%$ CI $54-77 \%)$ resulting in a good correlation $\left(\kappa_{\mathrm{w}}=0.73,95 \%\right.$ CI $0.64-0.83, p<0.0001$ ). The proportion of "unclear" readings was $6.8 \%$ in the paired reading vs $13.6 \%$ in the isolated reading. Adding the baseline PET scan altered the PET classification in 15 cases but in either direction of suspicion: in eight patients the level of suspicion increased, in seven decreasing (Table 2a). In six cases (13.6\%) the conclusion was opposite, with three false positive and three false negative (Fig. 1) isolated PET readings. Dichotomization did not clearly affect the results with accuracies of $75 \%$ (95\% CI 63-84\%) and 77\% (95\% CI 65-86\%) for sensitive and conservative reading strategies, respectively (kappa's: $0.49,95 \%$ CI $0.28-0.71$ vs $0.50,95 \%$ CI $0.26-0.73$, respectively). False positivity rates of the isolated posttreatment PET interpretations prevailed in $4 / 14$ (29\%, 95\% CI $14-51 \%)$ and $6 / 20(30 \%, 95 \%$ CI $16-48 \%)$ for conservative and sensitive readings, respectively (Table $2 b$ ).

The interobserver agreement of paired baseline and posttreatment evaluations was similar compared to the isolated PET evaluations (linear weighted kappa's: 0.64, $95 \%$ CI $0.46-0.82$ vs $0.67,95 \%$ CI $0.51-0.83$, respectively).

\section{Per Lesion Analysis}

As expected, the large majority of regions were FDG negative: in the consensus setting, $95 \%$ of both isolated posttreatment scans and paired readings (Table $3 \mathrm{a}$ ); of the nonnegative readings, $22 \%$ and $17 \%$ were classified as "unclear," respectively (NS). Twentyfour of the isolated posttherapy PET classifications altered after addition of the baseline scan (similar to the patient-based analysis), and the change was in either direction of suspicion (12 towards lower level). Dichotomization yielded kappa's of 0.75 (95\% CI $0.66-0.85)$ and 0.71 (95\% CI $0.63-0.81)$ for conservative and sensitive reading strategies, respectively. The false positivity rates of isolated PET readings were $17 \%(95 \% \mathrm{CI}$ 9-30\%) and $24 \%(95 \%$ CI $16-36 \%)$ with conservative and sensitive readings, respectively. The interobserver agreement for the per lesion evaluation of the isolated posttreatment PET scans was good $\left(\kappa_{\mathrm{w}}=0.71,95 \%\right.$ CI $\left.0.61-0.81, p<0.0001\right)$. Adding the baseline PET information did not further improve the observer agreement $\left(\kappa_{\mathrm{w}}=0.66,95 \%\right.$ CI $\left.0.52-0.75, p<0.0001\right)$. Discrepant lesional scores were randomly divided over the 22 different regions, the number of different scores ranging from 1 to 7 for each region in 20 of 22 regions.

\section{Discussion}

The addition of baseline to posttreatment PET evaluation affected the classification of metabolic response in $34 \%$ of malignant lymphoma patients treated with first-line chemotherapy. In one out of seven patients, addition of the baseline PET lead to opposite conclusions (95\% CI 4-14). False positivity was reduced by adding the baseline scan information, but the effect on false negativity was similar. In addition, the amount of unclear classifications reduced $(50 \%)$ after paired reading. Observer agreement did not improve upon adding the baseline PET data.

In malignant lymphoma, baseline PET information is essential to be able to assess response in lymphoma types with variable FDG avidity. In routinely FDG-avid lymphoma, baseline scans are recommended but not mandatory [8]. The intrinsic paradox flows from a lack of evidence, primarily at the level of effectiveness. Obvious disadvantages of routine baseline scanning are costs and unnecessary radiation (typically about $3.5 \mathrm{mSv}$ ) added by FDG to the standard CT work-up [12, 13]. In case of adding low-dose $\mathrm{CT}$ to PET, augmented with another 2-3 mSv [14]. Even though diagnostic CT (typically extending from neck to perineum in these patients) yields most of the radiation dose, adding PET should be justified by an impact on disease classification.

Our study has some limitations. First, PET scans in this study were performed with a stand-alone PET scanner rather than with PET-CT. Second, the observers were blinded for type and grade of lymphoma, pretreatment, or posttreatment situation and CT findings. An argument favoring interpreting diagnostic tests with clinical information is that the accuracy of the read may be improved by the additional information. It could be argued that the added value of the pretreatment PET scan may have been less, when this information had been provided. An argument favoring interpreting diagnostic tests without clinical information is that it may bias the reading and that clinical information should be incorporated into decision-making only after an unbiased read [15]. In three patients, one region which was initially not affected according to the clinical digital database was classified as equivocal in the first session. After pairedreading, these scores were changed into negative. In this situation the pretreatment scan provided the same information as the knowledge of the initial clinical information and instead of $34 \%(15 / 44), 27 \%(12 / 44)$ of the overall results differed.

In conclusion, without any other clinical information, a pretreatment PET facilitates and changes the interpretation of a posttreatment PET in a third of the patients, resulting in both upgrading and downgrading of the posttreatment situation of a malignant lymphoma patient. Adding a baseline PET scan did not improve observer agreement. If these results are confirmed for PET/CT systems, they favor the addition of baseline PET to the current work-up of patients with malignant lymphoma.

Open Access. This article is distributed under the terms of the Creative Commons Attribution Noncommercial License which permits any noncommercial use, distribution, and reproduction in any medium, provided the original author(s) and source are credited. 


\section{Appendix}

Table 4. Results per case of the PET evaluation

\begin{tabular}{|c|c|c|c|c|c|c|c|c|}
\hline \multirow[t]{2}{*}{ Cases } & \multirow[t]{2}{*}{ Initial stage and PA } & \multicolumn{3}{|c|}{ Post $^{\mathrm{a}}$} & \multicolumn{3}{|c|}{ Post and pre ${ }^{\mathrm{b}}$} & \multirow[t]{2}{*}{ Difference } \\
\hline & & $\mathrm{O}_{1}$ & $\mathrm{O}_{2}$ & $\mathbf{C}$ & $\mathrm{O}_{1}$ & $\mathrm{O}_{2}$ & $\mathbf{C}$ & \\
\hline 1 & 4, NHL; small lymphocytic lymphoma & + & + & + & + & + & + & No \\
\hline 2 & 2. NHL; diffuse large B cell lymphoma & $\mathrm{U}^{\mathrm{d}}$ & $\mathrm{U}$ & $\mathrm{U}$ & + & + & + & Yes \\
\hline 3 & 3, NHL; diffuse large B cell lymphoma & - & - & - & - & - & - & No \\
\hline 4 & $2, \mathrm{HD}$; nodular sclerosis & $\mathrm{U}$ & $\mathrm{U}$ & $\mathrm{U}$ & - & - & - & Yes \\
\hline 5 & 2, NHL; diffuse large B cell lymphoma & - & $\mathrm{U}$ & - & - & - & - & No \\
\hline 6 & 4, NHL; Burkitt & - & - & - & - & - & - & No \\
\hline 7 & 2. NHL; follicular & + & + & + & + & + & + & No \\
\hline 8 & 3, HD; nodular sclerosis & $\mathrm{U}$ & $\mathrm{U}$ & $\mathrm{U}$ & - & - & - & Yes \\
\hline 9 & 4, NHL; diffuse large B cell lymphoma & - & - & - & + & $\mathrm{U}$ & + & Yes \\
\hline 10 & 4, NHL; diffuse large B cell lymphoma & - & - & _- & - & - & - & No \\
\hline 11 & $1, \mathrm{HD}$; mixed cellularity & $\mathrm{U}$ & $\mathrm{U}$ & + & - & - & - & Yes \\
\hline 12 & 4, NHL; follicular & - & - & - & - & + & + & Yes \\
\hline 13 & 3, NHL; diffuse large B cell lymphoma & - & - & - & - & - & - & No \\
\hline 14 & 2, NHL; diffuse large B cell lymphoma & + & + & - & + & $\mathrm{U}$ & $\mathrm{U}$ & Yes \\
\hline 15 & 2. HD; nodular sclerosis & + & + & + & + & + & + & No \\
\hline 16 & 4, NHL; mucose-related lymphoid tissue & + & + & + & + & + & + & No \\
\hline 17 & 3. NHL; diffuse large B cell lymphoma & - & - & - & + & + & + & Yes \\
\hline 18 & 2, NHL; diffuse large B cell lymphoma & - & - & - & - & - & - & No \\
\hline 19 & 4, NHL; follicular & - & - & - & - & - & - & No \\
\hline 20 & 2, $\mathrm{HD}$; mixed cellularity & - & - & - & - & - & - & No \\
\hline 21 & $2, \mathrm{HD} ;$ nodular sclerosis & + & + & + & - & + & + & No \\
\hline 22 & 3, NHL; diffuse large B cell lymphoma & + & $\mathrm{U}$ & $\mathrm{U}$ & + & + & + & Yes \\
\hline 23 & 3, NHL; diffuse large B cell lymphoma & $\mathrm{U}$ & + & $\mathrm{U}$ & + & + & + & Yes \\
\hline 24 & 2, NHL; anaplastic cell lymphoma & + & $\mathrm{U}$ & + & $\mathrm{U}$ & + & + & No \\
\hline 25 & 3, NHL; diffuse large B cell lymphoma & - & $\mathrm{U}$ & $\mathrm{U}$ & - & - & - & Yes \\
\hline 26 & $4, \mathrm{HD}$; nodular sclerosis & - & - & - & - & - & - & No \\
\hline 27 & 3. NHL; diffuse large B cell lymphoma & + & + & + & + & + & + & No \\
\hline 28 & 3, NHL; follicular & + & + & + & - & + & + & No \\
\hline 29 & 1, NHL; diffuse large B cell lymphoma & - & - & - & - & - & - & No \\
\hline 30 & 3, NHL; diffuse large B cell lymphoma & - & - & - & - & - & - & No \\
\hline 31 & 3, NHL; mantle cell lymphoma & - & - & - & - & - & - & No \\
\hline 32 & 3. NHL; follicular & - & - & _- & - & - & - & No \\
\hline 33 & 2, NHL; follicular & - & - & - & - & - & - & No \\
\hline 34 & 2, HD; nodular sclerosis & - & - & - & - & - & - & No \\
\hline 35 & 4. HD; nodular sclerosis & $\mathrm{U}$ & - & - & - & - & - & No \\
\hline 36 & 3, NHL; diffuse large B cell lymphoma & - & + & + & + & + & + & No \\
\hline 37 & 3, NHL; mantle cell lymphoma & + & + & + & $\mathrm{U}$ & $\mathrm{U}$ & $\mathrm{U}$ & Yes \\
\hline 38 & 3, NHL; follicular & - & $\mathrm{U}$ & - & - & $\mathrm{U}$ & - & No \\
\hline 39 & 2, NHL; marginal zone lymphoma & + & + & + & + & + & + & No \\
\hline 40 & 3, NHL; small lymphocytic lymphoma & $\mathrm{U}$ & - & - & $\mathrm{U}$ & $\mathrm{U}$ & $\mathrm{U}$ & Yes \\
\hline 41 & 1, NHL; follicular & + & + & + & - & + & - & Yes \\
\hline 42 & 4. NHL; diffuse large B cell lymphoma & - & - & - & - & - & - & No \\
\hline 43. & 2, NHL; follicular & + & + & + & - & $\mathrm{U}$ & - & Yes \\
\hline 44 & 2, NHL; diffuse large B cell lymphoma & - & - & - & - & - & - & No \\
\hline
\end{tabular}

${ }^{\text {a}}$ Post: per patient evaluation of the posttreatment PET scan without knowledge of the pretreatment PET scan

${ }^{b}$ Post and pre: per patient evaluation of the posttreatment PET scan after visualization of the pretreatment PET scan, i.e., paired reading

${ }^{\mathrm{c}}$ Difference: if there is a difference in interpretation of the posttreatment PET with and without the knowledge of the pretreatment PET

${ }^{\mathrm{d}}$ Unclear: when one or more regions are scored equivocal and all other regions are classified negative in the PET scan, it is not possible to determine whether the scan is positive or negative, and the conclusion is unclear

\section{References}

1. Hoskin PJ (2003) PET in lymphoma: what are the oncologist's needs? Eur J Nucl Med Mol Imaging 30(Suppl 1):S37-S41

2. Carbone PP, Kaplan HS, Musshoff K et al (1971) Report of the committee on Hodgkin's disease staging classification. Cancer Res $31: 1860-1861$

3. Armitage JO (2005) Staging non-Hodgkin lymphoma. CA Cancer J Clin 55:368-376
4. Hoh CK, Glaspy J, Rosen P et al (1997) Whole-body FDG-PET imaging for staging of Hodgkin's disease and lymphoma. J Nucl Med 38:343-348

5. Buchmann I, Reinhardt M, Elsner K et al (2001) 2-(fluorine-18)fluoro2-deoxy-D-glucose positron emission tomography in the detection and staging of malignant lymphoma. A bicenter trial. Cancer 91:889-899

6. Seam P, Juweid ME, Cheson BD (2007) The role of FDG-PET scans in patients with lymphoma. Blood 110:3507-3516 
7. Juweid ME, Stroobants S, Hoekstra OS et al (2007) Use of positron emission tomography for response assessment of lymphoma: consensus of the imaging subcommittee of International Harmonization Project in Lymphoma. J Clin Oncol 25:571-578

8. Cheson BD, Pfistner B, Juweid ME et al (2007) Revised response criteria for malignant lymphoma. J Clin Oncol 25:579-586

9. Manning K, Tepfer B, Goldklang G et al (2007) Clinical practice guidelines for the utilization of positron emission tomography/computed tomography imaging in selected oncologic applications: suggestions from a provider group. Mol Imaging Biol 9:324-332

10. Lister TA, Crowther D, Sutcliffe SB et al (1989) Report of a committee convened to discuss the evaluation and staging of patients with Hodgkin's disease: cotswolds meeting. J Clin Oncol 7:1630-1636
11. Altman DG (1999) Inter-rater agreement. In: Altman DG (ed) Practical statistics for medical research. Chapman \& Hall, London, pp 403-409

12. $\mathrm{Wu} \mathrm{TH}$, Huang $\mathrm{YH}$, Lee JJ et al (2004) Radiation exposure during transmission measurements: comparison between CT- and germaniumbased techniques with a current PET scanner. Eur J Nucl Med Mol Imaging 31:38-43

13. Brix G, Lechel U, Glatting G et al (2005) Radiation exposure of patients undergoing whole-body dual-modality $18 \mathrm{~F}-\mathrm{FDG}$ PET/CT examinations. J Nucl Med 46:608-613

14. Kinahan PE, Townsend DW, Beyer T et al (1998) Attenuation correction for a combined 3D PET/CT scanner. Med Phys 25:2046-2053

15. Griscom NT (2002) A suggestion: look at the images first, before you read the history. Radiology 223:9-10 